\title{
WAVE-LENGTH MEASUREMENTS IN ARC SPECTRA PHOTOGRAPHED IN THE YELLOW, RED, AND INFRA-RED
}

\author{
By F. M. Walters, Jr.
}

\section{ABSTRACT}

For several years the Bureau of Standards has been conducting grating measurements in red and infra-red arc spectra by the aid of specially sensitized photographic plates to improve the data in this region and to find some element which would furnish lines suitable for wave-length standards in this region. To the 25 elements already measured the following are here added: Silver, aluminum, gold, bismuth, cadmium, mercury, lead, antimony, tin, and zinc.

The wave-length measurements are in the international system and are given to $0.01 \mathrm{~A}$.

The elements were brought to luminosity by inserting a sample in copper or graphite electrodes between which the arc was maintained. The grating used had 299 lines per millimeter and a $640-\mathrm{cm}$ radius. The spectra were photographed in the first order on plates sensitized with pinacyanol or dicyanin. The comparison spectrum was iron in the first, second, or third order, and in the reductions the wave lengths established by interference methods were used.

Impurity and spurious lines must be looked for very carefully. It is necessary to describe exactly the source of light and specify the observing conditions. The accuracy possible in wave-length measurements from the direct photography of normal spectra exceeds that in measurements from phosphor photography and bolometer measurements.

\section{CONTENTS}

Page

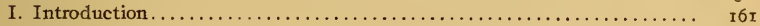

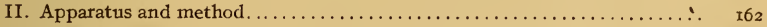

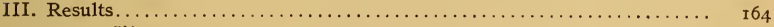

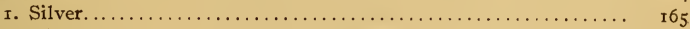

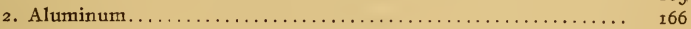

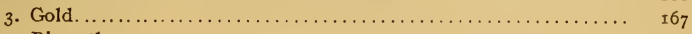

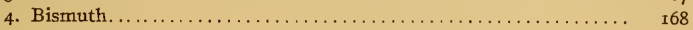

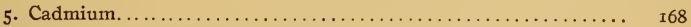

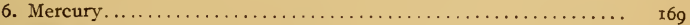

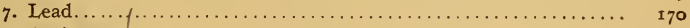

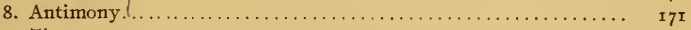

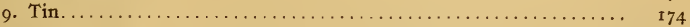

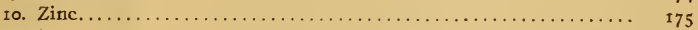

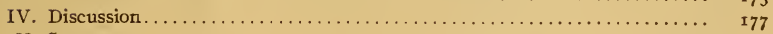

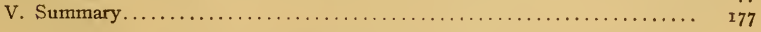

\section{INTRODUCTION}

Several years ago the Bureau of Standards began a program of grating measurements of the red and infra-red arc spectra of the chemical elements. The purpose was to find some element which 
would furnish lines suitable for wave-length standards in this region, and also to improve the data on arc spectra with the aid of specially sensitized photographic plates. Wave-length measurements in spectra obtained by photography have already been published for 25 elements, namely, lithium, sodium, potassium, rubidium, caesium, copper, beryllium, calcium, strontium, barium, and magnesium; ${ }^{1}$ iron, cobalt, and nickel; ${ }^{2}$ helium; ${ }^{3}$ neon; ${ }^{4}$ krypton and xenon; ${ }^{5}$ titanium, vanadium, chromium, manganese, molybdenum, tungsten, and uranium. ${ }^{6}$ The greater the number of elements which have been measured the easier it is to eliminate the impurity lines in the observed spectrum of the next. Only in this way can the true spectra which are characteristic of each element be ultimately determined.

This paper is a continuation of the program and presents wavelength measurements in international Angstrom units in the spectra of silver, aluminum, gold, bismuth, cadmium, mercury, lead, antimony, tin, and zinc from wave lengths $5500 \mathrm{~A}$ to about I0 $000 \mathrm{~A}$.

\section{APPARATUS AND METHOD}

The Anderson grating employed in this work-radius of curvature, $640 \mathrm{~cm}$, and 299 lines to the millimeter-is mounted in parallel light ${ }^{7}$ and has a dispersion in the first order of about Io A per millimeter.

Plates 8 inches long were used so that a wave-length interval of $2000 \mathrm{~A}$ was recorded in one exposure to the first order spectrum. The photographs were made on Seed's 23 and Seed's 30 plates sensitized to the long waves by staining in dye baths containing water, alcohol, and ammonia. The dye used, the time of exposure, the current in the arc, and slit width for the various regions are given in the following table:

\begin{tabular}{|c|c|c|c|c|}
\hline Region & Dye & Time & Current & $\begin{array}{c}\text { Slit } \\
\text { width }\end{array}$ \\
\hline $5500-7500 \mathrm{~A} \ldots \ldots$ & 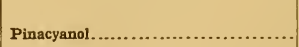 & $\begin{array}{r}\text { Minutes } \\
20-60\end{array}$ & $\begin{array}{c}\text { Amperes } \\
6.5\end{array}$ & $\begin{array}{l}\mathrm{mm} \\
0.025\end{array}$ \\
\hline $6400-8400$ A................... & 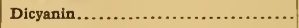 & $45-100$ & 7.5 & .035 \\
\hline $7300-9300$ A.................... & ..... do....... & $75-300$ & 8.5 & .045 \\
\hline $8300-10300 \mathrm{~A} . \ldots \ldots \ldots \ldots \ldots . . . . . .$. & .....do............ & 300 & $8-12$ & .055 \\
\hline
\end{tabular}

${ }^{1}$ B. S. Bull., 14, p. $371 ; 1917$

2 B. S. Bull., 14, p. 637 ; 1918.

${ }^{3}$ B. S. Bull., 14, p. 157; 1917.

B. S. Bull., 14, p. 77x; 1918.
B. S. Sci. Papers, 15, p. 251; 1919.

6 B. S. Sci. Papers, 16, p. 51; 1920.

7B.S. Bull., 14, p. 371; 1917. 
The overlapping second and third orders were screened out by a cell of potassium bichromate or a piece of Jena red glass.

On account of the relatively low melting points of most of the metals used in this work it was impossible to employ electrodes of the metals themselves, so they were brought to luminosity by inserting a sample in a hole bored in one of two copper or graphite terminals between which the arc was maintained. Copper rods about $7 \mathrm{~mm}$ and graphite rods $\mathrm{I} 2 \mathrm{~mm}$ in diameter were used with the positive electrode below, and the arc was fed with direct current from a power line of 240 volts. The average length of the arc was about $6 \mathrm{~mm}$, and light from the central portion was focused on the slit of the spectrograph by means of a lens so placed as to give a threefold magnification. The use of copper electrodes for spectrographic analysis offers certain advantages over the older practice of using electrodes of carbon or graphite, in that very pure copper can readily be obtained and its spectrum in the region of longer waves is comparatively simple, so that there is less chance of being confused with lines due to the electrodes and impurities. Spectroscopic analysis of the graphite employed in this work showed that the alkali metals, alkaline earths, and iron, titanium, and vanadium were present as impurities. Graphite electrodes were frequently used, especially in cases where it appeared that copper lines were nearly coincident with lines due to other elements and might thus interfere with the accurate measurement of the latter.

In either case, whether copper or graphite electrodes were used, the intensity of the spectrum of copper or of carbon and its impurities was somewhat reduced by keeping the positive electrode loaded with the metal under investigation, so that the arc stream was always charged with the vapor of the element. When this condition existed for the elements dealt with in this work, the arc made a loud hissing sound and also exhibited colors in core and aureole which are more or less characteristic for each of the metals. If the arc burned much more quietly and showed that only the undesirable spectrum of copper or of graphite was being produced, the circuit was broken, a new supply of metal was placed in the crater, and the arc was again struck. The relatively low boiling points of the metals under discussion and the rapidity with which most of them oxidize made it necessary to feed the arc at short intervals of time, so that in some cases 100 or more grams of metal were consumed in making a five-hour exposure. 
For the comparison spectrum electrodes of Norwegian or electrolytic iron were used under the conditions recommended by the International Wave Length Committee. ${ }^{8}$

The plates were measured on a large Gaertner engine, first with the scale reading increasing with the wave length, and then reversed, so as to eliminate personal error in setting on lines of different intensity and width. The iron in the first, second, or third order served as the reference spectrum, and in the reductions the wave lengths determined by interference methods ${ }^{9}$ were used.

Lines due to impurities were eliminated from the spectra by reference to Kayser's Handbuch, to the work already done at the Bureau of Standards on the spectra of other elements, and by intercomparison among the wave lengths of the elements observed in this paper. Faint lines common to two or more elements were removed even when they could not be ascribed to any known element. Among these the most persistent were $5727.96 \mathrm{~A}$, 6739.I I A, and $8184.86 \mathrm{~A}$. It is possible that these three are new lines of copper. Ghosts of both the Rowland and also the Lyman ${ }^{10}$ type were looked for and eliminated from the tables. The wave lengths were examined also for second order lines which might have passed through the screens used.

\section{RESULTS}

The first column of each of the following tables gives the wave lengths measured in international Angstrom units. The second column gives notes on the intensity and character of the lines. The strongest lines are given an intensity of 10 and the faintest measurable lines an intensity of $\mathrm{I}$. This estimate was applied to the work as a whole rather than to the spectrum of any particular element, so that a value of Io, for example, varied somewhat with the region under observation. To illustrate, with the methods employed $6438 \mathrm{~A}$ of cadmium gives a much greater photographic effect than $8273 \mathrm{~A}$ of silver, but an intensity of 10 is assigned to each of these lines in the tables. The character of a line is indicated by letters having the following meanings:

$$
\begin{aligned}
\mathrm{b} & =\text { broad. } \\
\mathrm{h} & =\text { hazy. } \\
\mathrm{H} & =\text { very hazy. } \\
\mathrm{L} & =\text { shaded to the red. } \\
\mathrm{v} & =\text { shaded to violet. }
\end{aligned}
$$


It is difficult to express definite wave lengths for lines which are unsymmetrical in structure; $i$. e., shaded to red or to violet. An effort was always made to set on the "center of gravity" of the image, but in the case of unsymmetrical lines this setting becomes a function of the photographic exposure. For lines shaded to the red a strongly exposed spectrogram will thus give longer wave lengths than those obtained from weaker spectrograms. The uncertainty in expressing the wave lenghts of unsymmetrical lines may be several hundredths of an Angstrom on this account. The third column indicates the probable error of the measurements of each wave length, the significance of the letters being as follows:

$$
\begin{aligned}
& \mathrm{A}=\text { probable error } 0.01 \mathrm{~A} . \\
& \mathrm{B}=\text { probable error of } 0.01 \mathrm{~A} \text { to } 0.02 \mathrm{~A} . \\
& \mathrm{C}=\text { probable error of } 0.02 \mathrm{~A} \text { to } 0.03 \mathrm{~A} . \\
& \mathrm{D}=\text { probable error }>0.03 \mathrm{~A} . \\
& \mathrm{E}=\text { only one determination. }
\end{aligned}
$$

The wave-length measurements of other observers are included in the tables for the purpose of comparison. Whenever these wave lengths are expressed in international Angstrom units it is so indicated by I. A. in the table headings. The remaining values are based on Rowland's system of wave-length standards and may be changed to the international system by subtracting the following quantities:

$$
\begin{aligned}
& 0.22 \mathrm{~A} \text { from } 5500 \text { to } 6050 \mathrm{~A} \text {. } \\
& .2 \mathrm{I} \text { A from } 6050 \text { to } 6500 \mathrm{~A} \text {. } \\
& .22 \mathrm{~A} \text { from } 6500 \text { to } 6570 \mathrm{~A} \text {. } \\
& .23 \mathrm{~A} \text { from } 6570 \text { to } 6750 \mathrm{~A} \text {. } \\
& .24 \mathrm{~A} \text { from } 6750 \text { to } 6850 \mathrm{~A} \text {. } \\
& .25 \mathrm{~A} \text { from } 6850 \text { to } 7000 \mathrm{~A} \text {. } \\
& .26 \mathrm{~A} \text { from } 7000 \text { to } 7200 \mathrm{~A} \text {. } \\
& .27 \mathrm{~A} \text { from } 7200 \text { to } 7400 \mathrm{~A} \text {. } \\
& .28 \mathrm{~A} \text { from } 7400 \text { to } 7700 \mathrm{~A} \text {. } \\
& .29 \mathrm{~A} \text { from } 7700 \text { to } 8000 \mathrm{~A} \text {. } \\
& .30 \mathrm{~A} \text { from } 8000 \text { to } 8200 \mathrm{~A} \text {. } \\
& .31 \mathrm{~A} \text { from } 8200 \text { to } 8300 \mathrm{~A} \text {. } \\
& \text { about } .35 \mathrm{~A} \text { from } 8300 \text { to } 8800 \mathrm{~A} \text {. }
\end{aligned}
$$

\section{SILVER}

Metallic silver and silver nitrate were burned in copper. Nineteen plates were measured; three of them were 5 -hour exposures in the region $8400-10400 \mathrm{~A}$, but no wave lengths greater than 8273.58 A were found. 
TABLE 1.-Silver

\begin{tabular}{|c|c|c|c|c|c|c|c|c|}
\hline \multicolumn{3}{|l|}{ Walters } & \multicolumn{2}{|c|}{ Kaspar a } & \multicolumn{2}{|c|}{$\begin{array}{c}\text { Kayser and } \\
\text { Runge } b\end{array}$} & \multicolumn{2}{|c|}{ Lehmann $c$} \\
\hline$\lambda I . A$. & Notes & $\begin{array}{c}\text { Probable } \\
\text { error }\end{array}$ & $\lambda \mathbf{I} . \mathbf{A}$. & Notes & $\lambda$ & Notes & $\lambda$ & Notes \\
\hline $5523.74 \ldots \ldots$ & 3 & B & & & & & & \\
\hline $5529.91 \ldots \ldots \ldots \ldots \ldots . . . . . . .$. & 2 & $\mathbf{A}$ & …. & & (n.......... & ... & . & - \\
\hline $5545.67 \ldots \ldots \ldots \ldots \ldots \ldots \ldots$ & $3, I$ & $\mathbf{B}$ & 5545.635 & & 5545.86 & 4 & ........ & ......... \\
\hline \multirow[t]{3}{*}{$5667.34 \ldots \ldots \ldots \ldots \ldots \ldots \ldots \ldots$} & $4, \mathrm{~h}$ & B & 5667.494 & (......... & 5667.72 & 4 & -..... & (n....... \\
\hline & & & \multicolumn{2}{|c|}{ Randall $d$} & \multicolumn{2}{|c|}{ Lewis e } & & \\
\hline & & & $\lambda$ & Notes & $\lambda$ & Notes & & \\
\hline $7687.79 \ldots \ldots$. & 10 & B & 7688.2 & 200 & 7688.4 & & 7684.0 & 1 \\
\hline $8273.58 \ldots \ldots \ldots \ldots . . .$. & 10 & C & 8274.1 & 250 & 8274.08 & & 8275.4 & 1 \\
\hline
\end{tabular}

a Kaspar, Zs. für wissenschaftliche Photographie, 10, p. 53; 1912.

$b$ Kayser and Runge, Wied. Ann., 46, p. 225; 1892.

cLehmann, Ann. d. Phys., 39, p. 53; 1912 (phosphor photography).

$d$ Randall, Astrophys. J., 43, p. I; IgII (bolometer measurements).

$e$ Lewis, Astrophys. J., 2, p. I; 1895 (bolometer measurements).

Eder (Wien, Ber., 124; I915) gives the wave length of the deep red line as 7687.89 and recommends its use in place of the red doublet of potassium for the calibration of small spectroscopes.

\section{ALUMINUM}

The commercial metal was burned in copper or graphite. Some observations were made with the positive electrode aluminum, but with this arrangement a current of more than 5 amperes could not be used without melting the aluminum.

TABLE 2.-Aluminum

\begin{tabular}{|c|c|c|c|c|c|c|c|c|c|c|c|c|}
\hline \multicolumn{3}{|c|}{ Walters } & \multicolumn{2}{|c|}{ Paschen a } & \multicolumn{2}{|c|}{$\begin{array}{l}\text { Eder and } \\
\text { Valenta } b\end{array}$} & \multicolumn{2}{|c|}{ Grinter c } & \multicolumn{2}{|c|}{ Eder $d$} & \multicolumn{2}{|c|}{ Lehmann $e$} \\
\hline$\lambda$ I. A. & Notes & $\begin{array}{l}\text { Prob- } \\
\text { able } \\
\text { error }\end{array}$ & $\lambda$ & Notes & $\lambda$ & Notes & $\lambda$ I. A. & Notes & $\lambda$ I. A. & Notes & $\lambda$ & Notes \\
\hline 5557.02 & 3 & D & 5557.283 & 7 & 5557.57 & & 5557.08 & $1, \mathrm{u}$ & & & & \\
\hline 5557.96 & 3 & $\mathrm{D}$ & 5558.167 & 5 & 5558.37 & & 5557.95 & $1, u$ & & & & \\
\hline 5635.15 & 2 & D & & & & & & & & & & \\
\hline 5651.69 & $2, \mathrm{~h}$ & B & & & & & & & & & $\ldots$ & ... \\
\hline 6176.36 & $2, \mathrm{~h}$ & $\bar{D}$ & & & & & & & & & & \\
\hline 6696.12 & 7 & $\mathbf{A}$ & 6696.269 & & & & 6696.064 & 3 & 6696.08 & 5 & ....... & …..... \\
\hline 6698.78 & 5 & $\mathbf{A}$ & 6698.936 & & & & & 3 & 6698.77 & 4 & & \\
\hline 7362.5 & 2, H, I & D & & & & & & & & & & \\
\hline
\end{tabular}

a Paschen, Ann. d. Phys. (4), 29, p. 625; 1909.

$b$ Eder and Valenta, Atlas Typischer Spectren, Wien; rgrr.

${ }^{c}$ Grünter, Zs. f. wiss. Phot., 13, p. 11; $191^{-14}$.

dEder, Wien, Ber.; r9r5.

e Lehmann, Ann. d. Phys., 39, p. 53; 1912. 
TABLE 2-Continued

\begin{tabular}{|c|c|c|c|c|c|c|c|c|c|c|c|c|}
\hline \multicolumn{3}{|c|}{ Walters } & \multicolumn{2}{|c|}{ Paschen } & \multicolumn{2}{|c|}{ Melssner $a$} & \multicolumn{2}{|c|}{ Grïnter } & \multicolumn{2}{|c|}{ Eder } & \multicolumn{2}{|c|}{ Lehmann } \\
\hline$\lambda I . A$. & Notes & $\begin{array}{l}\text { Prob- } \\
\text { able } \\
\text { error }\end{array}$ & $\lambda$ & Notes & $\lambda$ I. A. & Notes & $\lambda$ I. A. & Notes & $\lambda$ I. A. & Notes & $\lambda$ & Notes \\
\hline 7837.1 & $6, \mathrm{H}, \mathrm{L}$ & D & & & $\begin{array}{l}7466 \\
7836.85\end{array}$ & $\begin{array}{l}3, \mathrm{u} \\
5\end{array}$ & & & & & & \\
\hline 8774.48 & $6, \mathbf{L}$ & C & 8775.1 & 50 & 8774.56 & 4 & & & & & $\begin{array}{l}7833.5 \\
8770.1\end{array}$ & $\begin{array}{l}2 \\
2\end{array}$ \\
\hline
\end{tabular}

a Meissner, Ann. d. Phys., 50, p. 7r3; rgr6.

\section{GOLD}

Gold chloride or metallic gold was burned in copper or graphite. Thirteen plates were measured. Silver was the principal impurity. The lines 6122 and $6162 \mathrm{~A}$ observed by Quincke are in close agreement with strong calcium lines.

TABLE 3.-Gold

\begin{tabular}{|c|c|c|c|c|c|c|c|c|}
\hline \multicolumn{3}{|l|}{ Walters } & \multicolumn{2}{|c|}{ Quincke $a$} & \multicolumn{2}{|c|}{$\begin{array}{l}\text { Eder and } \\
\text { Valenta } b\end{array}$} & \multicolumn{2}{|c|}{$\begin{array}{l}\text { Kayser and } \\
\text { Runge } c\end{array}$} \\
\hline$\lambda$ I. A. & Notes & $\begin{array}{c}\text { Probable } \\
\text { error }\end{array}$ & $\lambda$ I. A. & Notes & $\lambda$ & Notes & $\lambda$ & Notes \\
\hline $5629.27 \ldots$ & $1, \mathbf{H}$ & $\mathbf{D}$ & & & & & & \\
\hline $5651.07 \ldots \ldots \ldots \ldots$. & 1 & D & (n............ & ........ & (n.......... & .... & $\ldots \ldots$ & ..... \\
\hline $5655.79 \ldots \ldots \ldots \ldots$ & 3 & C & 5655.764 & 1 & 5656.00 & 4 & 5656.00 & 4 \\
\hline $5660.46 \ldots$ & 1 & D & ..... & .... & ….......... & & ........... & -. \\
\hline $5837.40 \ldots \ldots \ldots \ldots . . . .$. & 5 & $\mathbf{A}$ & 5837.396 & 4 & 5837.64 & 10 & 5837.64 & 6 \\
\hline $5841.44 \ldots \ldots \ldots \ldots$ & 2 & B & .............. & .......... & .......... & & (n......... & ......... \\
\hline $5859.34 \ldots$ & $1, \mathbf{h}$ & D & (............. & ....... & ........... & & .......... & .. \\
\hline $5862.92 \ldots \ldots \ldots \ldots \ldots \ldots$ & 2 & $\mathbf{A}$ & 5862.943 & $1, \mathrm{u}$ & 5863.17 & 4 & 5863.17 & 6 \\
\hline $5956.98 \ldots \ldots \ldots \ldots \ldots \ldots \ldots$ & 3 & $\mathbf{B}$ & 5956.984 & $1, \mathrm{u}$ & 5957.24 & 5 & 5957.24 & 4 \\
\hline $5962.72 \ldots$. & 2 & $\mathbf{A}$ & 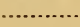 & & & & & -. \\
\hline \multirow[t]{4}{*}{$5968.96 \ldots \ldots \ldots \ldots \ldots \ldots$} & $1, \mathrm{H}$ & D & ............. & ....... & $\cdots$ & & $\cdots$ & .......... \\
\hline & & & 6101.654 & 2 & & & & \\
\hline & & & 6122.28 & $1, \mathrm{u}$ & (n............ & & 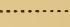 & $\cdots$ \\
\hline & & & 6162.228 & 3 & n......... & $\cdots$ & (n............. & …...... \\
\hline $6278.20 \ldots \ldots \ldots \ldots \ldots$ & 6 & $\mathbf{A}$ & 6279.179 & 4 & 6278.37 & 8 & 6278.37 & 4 \\
\hline \multirow[t]{2}{*}{ 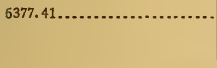 } & $1, \mathrm{~h}$ & D & (.............. & & & & & -. \\
\hline & & & 6634.22 & $1, \mathrm{u}$ & $\cdots$ & & & (n...... \\
\hline $6781.97 \ldots \ldots \ldots \ldots$ & $2, \mathrm{~h}$ & B & & $\ldots$ & & & & (n.......... \\
\hline $7023.28 \ldots \ldots$. & $1, \mathrm{~h}$ & $\mathbf{B}$ & & & 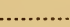 & & & ... \\
\hline \multirow[t]{3}{*}{$7156.82 \ldots \ldots \ldots$} & 1 & $\mathbf{A}$ & $\cdots$ & & ....... & & & .. \\
\hline & & & & & \multicolumn{2}{|c|}{ Eder $d$} & \multicolumn{2}{|c|}{ Lehmann e } \\
\hline & & & & & $\lambda$ I. A. & Notes & $\lambda$ & Notes \\
\hline $7510.69 \ldots$ & 5 & C & & & 7510.74 & 5 & 7509.8 & 1 \\
\hline
\end{tabular}

a Quincke Zeits. f. wiss., Phot., 14, p. 257; Igr4.

$b$ Eder and Valenta, A tlas Typischer Spectren, Wien; rgrr.

$c$ Kayser and Runge, Wied. Ann., 46, p. 225; r892.

$d$ Eder, Wien, Ber.; rgrs.

e Lehmann, Ann. d. Phys., 39, p. 53; rgr2.

$$
38351^{\circ}-21-2
$$




\section{BISMUTH}

Chemically pure metallic bismuth was burned in copper or graphite. Fourteen plates were measured. The principal impurities detected spectroscopically were silver and lead. The lines 8501.8 and $8627.9 \mathrm{~A}$ are very diffuse and are more than five Angstroms wide on the spectrograms.

TABLE 4.-Bismuth

\begin{tabular}{|c|c|c|c|c|c|c|c|c|c|c|}
\hline \multicolumn{3}{|c|}{ Walters } & \multicolumn{2}{|c|}{$\begin{array}{l}\text { Eder and } \\
\text { Valenta } a\end{array}$} & \multicolumn{2}{|c|}{$\begin{array}{c}\text { Kayser and } \\
\text { Runge } b\end{array}$} & \multicolumn{2}{|c|}{ Lehmann $c$} & \multicolumn{2}{|c|}{ Randall d } \\
\hline$\lambda$ I. A. & Notes & $\begin{array}{c}\text { Probable } \\
\text { error }\end{array}$ & $\lambda$ & Notes & $\lambda$ & Notes & $\lambda$ & Notes & $\lambda$ & Notes \\
\hline 5552.35 & $10, \mathrm{~B}$ & C & 5552.43 & 5 & 5552.43 & $8, r$ & & & & \\
\hline 5599.41 & 3 & A & & 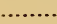 & & & & & & \\
\hline e5718. 81 & 2 & E & .......... & & ….. & $\cdots$ & & & & \\
\hline 5742.55 & 6 & B & 5742.77 & 1 & 42.74 & $4, x$ & & & & \\
\hline 6134.82 & 6 & B & 6135.08 & 4 & & & & & & \\
\hline 6184.99 & 2, H & D & & & & & & & & \\
\hline 6364.75 & $1, \mathrm{~h}$ & C & & & & & & & & .. \\
\hline 6475.73 & 3 & C & & & & & & & & \\
\hline 6476.24 & 3 & B & & & & & & & & \\
\hline 6991.12 & 4, H & C & & & & & & & & .. \\
\hline 7036.15 & 2 & B & & & & & & & & \\
\hline 7335.01 & 1 & D & & & & & & & & \\
\hline 7441.25 & $1, \mathrm{~h}$ & D & & & & & & & & \\
\hline 7502.33 & 2 & C & & & & & & & ... & ... \\
\hline 7838. 70 & 3 & B & & & & & & .. & 7841.1 & 7 \\
\hline 7840.33 & 2 & D & & & & & & & ..... & $\cdots$ \\
\hline 8210.83 & $1, \mathrm{~h}$ & D & & & & & 8205.5 & 4 & .... & $\cdots$ \\
\hline & & & & & & & 8343. 2 & 4 & $\ldots$. & \\
\hline 8501.8 & $1, \mathrm{H}, \mathrm{b}$ & D & & & & & 8508.3 & $4, u$ & .. & \\
\hline 8544.54 & 2 & $\mathbf{A}$ & .. & & & & ... & ...... & n.... & ....... \\
\hline 8579.74 & 1 & B & & & & & ......... & ........ & ........... & $\ldots .$. \\
\hline 8627.9 & 1, H, b & D & & & & & 8621.4 & 4 & 8628.5 & 10 \\
\hline 8754.88 & 2 & C & & & & & 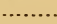 & $\cdots$ & & ....... \\
\hline 8761.54 & 3 & D & & & & & 8763.3 & 3 & 8761.8 & 10 \\
\hline 8907.81 & 2 & D & & & & & ......... & $\cdots$ & 8910.0 & 10 \\
\hline 9058.62 & 1 & D & & & & & 9054.4 & 3 & 9059.5 & 15 \\
\hline 9342.60 & $1, \mathrm{~h}$ & D & & & & & 9343.1 & 3 & 9344.1 & 40 \\
\hline 9657.20 & 2 & C & & & & & 9646.9 & 1 & 9657.9 & 300 \\
\hline
\end{tabular}

a Eder and Valenta, Atlas Typischer Spectren.

$b$ Kayser and Runge, Wied. Ann., 52, p. 93; 1894.

c Lehmann, Ann. d. Phys., 39, p. 53; rgre (phosphor photography).

$d$ Randall, Astrophys. J., 34, p. r; 19 rr (bolometer measurements).

e Given as spark line by W. Schwetz, Dissert., Bonn; 1908.

\section{CADMIUM}

Metallic cadmium was burned in copper or graphite electrodes. Fourteen plates were measured. Lead was found as an impurity. The lines measured by Paschen, 5599 to $6330 \mathrm{~A}$, were observed in cadmium arc in vacuo, and this may account for the absence of 
some of them in the present observations. It should be stated, however, that the first three lines were observed as a hazy group on spectrograms of the arc operated at atmospheric pressure, but the lines were too broad and poorly resolved to permit satisfactory measurements of wave lengths. The lines 6099, 6III, and 6I I $6 \mathrm{~A}$ constitute a similar group, and it is seen that they are very hazy, unsymmetrical, and of greater effective wave length when the arc is surrounded by atmospheric pressure instead of vacuum.

TABLE 5.-Cadmium

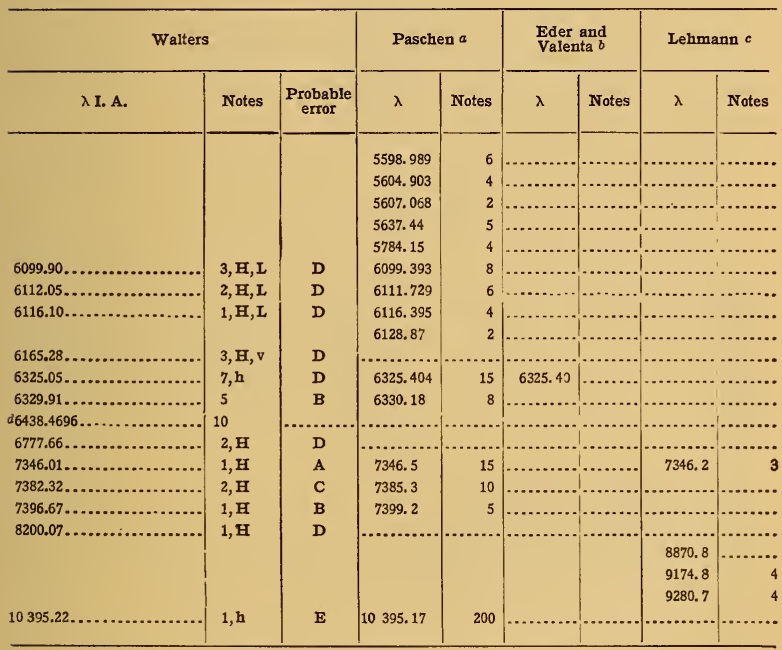

a Paschen, Ann. d. Phys., 29, p. 625.

$\checkmark$ E,der and Valenta, Atlas Typischer Spectren, Wien; rgx.

c Lehmann, Ann. d. Phys., 39, p. 53; 1912.

$d$ International value.

\section{MERCURY}

A vacuum arc in glass taking 3 amperes was used as the source. Exposures of 30 hours were made for the region beyond $8000 \mathrm{~A}$. The arc in air was tried but rejected on account of the broadness of the lines.

Wiedmann measured 5 lines of intensity 2 or less in prismatic spectra in the region 6878 to $8198 \mathrm{~A}$ which were too weak to photograph with a grating. 
TABLE 6.-Mercury

\begin{tabular}{|c|c|c|c|c|c|c|c|c|c|c|}
\hline \multicolumn{3}{|c|}{ Walters } & \multicolumn{2}{|c|}{ Styles $a$} & \multicolumn{2}{|c|}{ Wiedmann $b$} & \multicolumn{2}{|c|}{$\begin{array}{l}\text { Eder and } \\
\text { Valenta } c\end{array}$} & \multicolumn{2}{|c|}{$\begin{array}{l}\text { Kayser and } \\
\text { Runge } d\end{array}$} \\
\hline$\lambda$ I. A. & Notes & $\begin{array}{c}\text { Probable } \\
\text { error }\end{array}$ & $\lambda \mathrm{I} . \mathrm{A}$. & Notes & $\lambda$ & Notes & $\lambda$ & Notes & $\lambda$ & Notes \\
\hline 5676.01 & 3 & $\mathrm{D}$ & 5675.811 & 1 & & & 5676.01 & 2 & & \\
\hline \multicolumn{11}{|l|}{ e5769. 5984} \\
\hline & & & 5789.521 & 2 & & & & & & \\
\hline \multicolumn{11}{|l|}{$e 5790.6543$} \\
\hline \multirow[t]{2}{*}{5803.65} & 3 & C & & & & $\ldots$ & 5804.28 & 4 & 5804.28 & $2, r$ \\
\hline & & & & & & & & & 5819.05 & $1, \mathrm{u}$ \\
\hline \multirow[t]{2}{*}{ 5859. 38} & 3 & $\mathbf{A}$ & 5859.49 & $1, \mathrm{u}$ & 5860.28 & 1 & 5859.90 & 2 & & $\ldots .$. \\
\hline & & & & & 5868.28 & 1 & .......... & ... & & ..... \\
\hline 5872.03 & 1 & $\mathbf{E}$ & ............ & ..... & 5872.37 & 2 & 5872.78 & 2 & 5871.301 & $3, u$ \\
\hline 6072.64 & 2 & $\mathbf{A}$ & 6072.66 & 1 & 6072.839 & 5 & 6072.93 & 2 & & .... \\
\hline 6123.48 & 3 & A & 6123.48 & 1 & 6123.672 & 6 & 6123.65 & 2 & & \\
\hline \multirow[t]{5}{*}{6234.36} & 4 & $\mathbf{A}$ & 6234.31 & 2 & 6234.536 & 8 & ............. & $\cdots$ & & \\
\hline & & & & & & & & & \multicolumn{2}{|c|}{ Lehmann $f$} \\
\hline & & & & & & & & & $\lambda$ & Notes \\
\hline & & & & & & & & & 6521.1 & 3 \\
\hline & & & & & & & & & 6553.3 & $3, u$ \\
\hline 6716.34 & 4 & B & 6716.315 & 1 & 6716.680 & 5 & 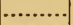 & $\ldots$ & .......en. & .......... \\
\hline 6907.46 & 8 & D & 6907.47 & 4 & 6907.776 & 10 & 6908.13 & 4 & 6917.03 & 3 \\
\hline 7081.94 & 4 & D & 7081.96 & 1 & 7082.273 & 4 & 7082.43 & 3 & 7085.7 & $4, u$ \\
\hline 7091.87 & 2 & D & ........... & ......... & 7092.456 & 3 & 7092.32 & 2 & 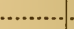 & ......... \\
\hline 7370.06 & 2 & $\mathbf{E}$ & ........... & ..... & 7371.00 & 3 & $\cdots$ & & & ... \\
\hline 7728.85 & 2 & C & ............. & .......... & 7729.456 & $\mathrm{u}$ & & & & ... \\
\hline 8195.60 & 1 & D & ............ & …..... & 8198 & 2 & .... & & & .......... \\
\hline
\end{tabular}

a Styles, Astrophys. J., 30, p. 48; 1909.

$b$ Wiedmann, Ann. d. Phys., 38, p. rogr; 1912.

c Ėder and Valenta, Atlas Typischer Spectren, Wien; rgrr.

d Kayser and Runge, Wied. Ann., 43, p. 384; 1891.

e Interferometer Measurements, Fabry and Perot, Comptes Rendus, 170, p. 492; 1900.

$f$ Lehmann, Ann. d. Phys., 39, p. 53; rgr2.

\section{LEAD}

The arc spectrum was obtained from ordinary metallic lead burning in copper electrodes or in graphite electrodes.

Eder states that the two red lines which he gives are reproauced in the Atlas Typischer Spectren, Chart XXI, No. 13. Although this reproduction shows 7229 with considerable intensity, no lines of longer wave length appear and the wave length $7700.18 \mathrm{~A}$, if real, is probably not due to lead.

The line 5895.70 A was measured in the fourth order. With low dispersion its presence is indicated by the increased intensity of the $\mathrm{D}_{1}$ line of sodium which usually occurs as an impurity. 
TABLE 7.-Lead

\begin{tabular}{|c|c|c|c|c|c|c|c|c|}
\hline \multicolumn{3}{|l|}{ Walters } & \multicolumn{2}{|c|}{$\begin{array}{l}\text { Eder and } \\
\text { Valenta } a\end{array}$} & \multicolumn{2}{|c|}{$\begin{array}{c}\text { Kayser and } \\
\text { Runge } b\end{array}$} & \multicolumn{2}{|c|}{ Lehmann $c$} \\
\hline$\lambda \mathbf{I} . \mathrm{A}$. & Notes & $\begin{array}{c}\text { Probable } \\
\text { error }\end{array}$ & $\lambda$ & Notes & $\lambda$ & Notes & $\lambda$ & Notes \\
\hline$d 5608.85 \ldots$ & 4 & $\mathbf{B}$ & 5609.14 & 1 & & & & \\
\hline $5692.26 \ldots$ & 4 & B & 5692.36 & 2 & & & & ....... \\
\hline $5895.70 \ldots$ & $6, \mathrm{~h}, \mathrm{~L}$ & B & 5896. 18 & $2+\mathrm{Na}$ & ....... & 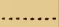 & & \\
\hline 6002.24. & $8, \mathrm{~h}, \mathrm{~L}$ & $\mathbf{D}$ & 6002.20 & $8, r$ & 6002.08 & $2, \mathrm{r}$ & & \\
\hline $6011.98 \ldots$ & $5, \mathrm{~h}, \mathrm{~L}$ & C & 6012.36 & 3,1 & & & & \\
\hline $6059.71 \ldots \ldots$ & $4, \mathrm{~h}, \mathrm{~L}$ & $\mathbf{B}$ & .......... & ...... & & & & \\
\hline $6110.70 \ldots$ & $3, \mathrm{~h}, \mathrm{~L}$ & $\mathbf{B}$ & 6111.32 & $2, \mathrm{r}$ & & & & \\
\hline $6169.40 \ldots \ldots \ldots \ldots$ & $2, \mathrm{~h}$ & $\mathrm{C}$ & 6169.66 & $2, u$ & & & & 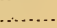 \\
\hline $6235.44 \ldots \ldots \ldots \ldots \ldots \ldots$ & 4 & B & 6235.73 & ....... & & & & \\
\hline \multirow[t]{3}{*}{$d 6660.17 .}$. & $3, \mathrm{~h}$ & B & n.......... & ....... & & & & .... \\
\hline & & & 6791.54 & $1, u$ & & & & $\cdots$. \\
\hline & & & 6878.67 & $1, \mathrm{u}$ & & & & \\
\hline \multirow[t]{3}{*}{$7099.78 \ldots \ldots \ldots \ldots \ldots \ldots \ldots \ldots$} & 2 & C & (n.......... & ......... & & & & ........ \\
\hline & & & \multicolumn{2}{|c|}{ Edere } & & & & \\
\hline & & & AI. A. & Notes & & & & \\
\hline $7229.11 \ldots$. & 10 & $\mathrm{C}$ & 7228.93 & 6 & & & 7228.6 & 1 \\
\hline $7330.12 \ldots$ & 2 & D & ............. & & & & & .......... \\
\hline & & & 7700.18 & 5 & & & & .......... \\
\hline $8272.85 \ldots$ & $3, \mathrm{~h}$ & C & 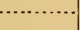 & & & & 8267.2 & $3 N$ \\
\hline
\end{tabular}

a Eder and Valenta, Wien, Ber., 119, IIa, p. 519; r9ro.

$b$ Kayser and Runge, Wied. Ann., 52, p. 93; 1894.

c Lehmann, Ann. d. Phys., 39, p. 53; r9r2.

$d$ Given by Klein as spark lines, Zeits. f. wiss. Phot., 12, p. 16; 191 3.

e Edder, Wien, Ber., rgrs.

\section{ANTIMONY}

Two samples of antimony were used. One was Kahlbaum pure metal and the other was less pure, containing lead, tin, calcium, sodium, potassium, cadmium, and iron. Twenty plates were measured. Five lines were observed which are given by Kretzer as occurring in the spark but not in the arc spectrum. The lines given by Kretzer beyond $7000 \mathrm{~A}$ are probably second order lines, since he used a potassium bichromate screen and a commercial panchromatic plate not particularly sensitive in this region. $7145.840,7279.591,7367.408$, and $7480.194 \mathrm{~A}$ correspond to the lead lines $3572.95,3639.72,3683.62$, and $3740.20 \mathrm{~A}$, which are the strongest in this region. $7276.08 \mathrm{I}$ and $7445.895 \mathrm{~A}$ correspond to the strongest antimony lines, 3638.00 and $3822.94 \mathrm{~A}$. Two lines 5700 and $5782 \mathrm{~A}$ observed by Schippers and by Kretzer are probably due to the presence of copper as an impurity. The sign + in the column of notes referring to my wave lengths indicates that these lines were very much stronger near the positive electrode than in any other part of the arc. 
TABLE 8.-Antimony

\begin{tabular}{|c|c|c|c|c|c|c|c|c|}
\hline \multicolumn{3}{|c|}{ Walters } & \multicolumn{2}{|c|}{ Eder and Valenta $a$} & \multicolumn{2}{|c|}{ Schippers $b$} & \multicolumn{2}{|c|}{ Kretzer $c$} \\
\hline$\lambda$ I. A. & Notes & $\begin{array}{c}\text { Probable } \\
\text { error }\end{array}$ & $\lambda$ & riotes & $\lambda$ I. A. & Notes & $\lambda$ & Notes \\
\hline $5531.89 \ldots$ & $1, \mathrm{~h}$ & D & & & & & & \\
\hline $5556.21 \ldots$ & 3 & B & 5556.37 & 2 & 5556.084 & 2 & & \\
\hline $5568.09 \ldots$ & 3 & B & …...... & $\cdots$ & 5567.963 & 1 & ............ & . \\
\hline $5599.93 \ldots .$. & 3 & B & 5600.05 & 2 & 5599.762 & 1 & $\ldots \ldots$. & \\
\hline $5602.30 \ldots$ & 3 & $\mathbf{B}$ & 5602.37 & 2 & 5602.113 & 2 & $\cdots$ & \\
\hline $5631.97 \ldots$ & 5 & c & & & ......... & & .. & \\
\hline $5639.74 \ldots$ & $2, \mathrm{~h}$ & A & & & ........... & ... & ........ & \\
\hline $5660.78 \ldots$ & $2, \mathrm{~h}$ & A & & & 5660.778 & 1 & . & .. \\
\hline $5691.26 \ldots$ & 2 & $\mathbf{A}$ & 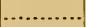 & & 5691.071 & 2 & 5691.367 & 4 \\
\hline & & & & & 5700.29 & 0 & 5700.439 & 3 \\
\hline $5707.47 \ldots$ & 2 & $\mathbf{A}$ & 5707.52 & 2 & 5707.494 & 2 & 5707.700 & 5 \\
\hline $5730.46 \ldots$ & 4 & B & 5730.56 & & 5730.314 & 2 & 7730.517 & 6 \\
\hline & & & & & 5774.557 & 3 & 5774.769 & 3 \\
\hline & & & & & 5782.150 & 3 & 5782. 299 & 4 \\
\hline $5806.30 \ldots$ & $1, \mathrm{~h}$ & D & & & ........... & ... & ............. & . \\
\hline & & & & & 5813.97 & 0 & 5814.040 & 2 \\
\hline $5844.66 \ldots$ & $1, \mathrm{H}$ & D & & & ……... & & ……...... & .... \\
\hline $5910.64 \ldots$ & $1, \mathrm{~h}, \mathrm{v},+$ & D & & & …....... & ... & ……...... & .... \\
\hline $5912.33 . \ldots \ldots \ldots \ldots \ldots$ & 2 & c & & & 5912.160 & 2 & 5912.357 & 5 \\
\hline $5948.37 \ldots$. & 1 & D & & & ............ & ... & ............. & .. \\
\hline$d 6005.00 \ldots . . . . . . .$. & $4, h,+$ & B & & & n......... & .. & 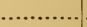 & . \\
\hline$d 6079.55 \ldots$ & $3, \mathrm{H},+$ & C & & & ............ & $\cdots$ & $\ldots \ldots \ldots .$. & . \\
\hline & & & & & 6081.14 & 0 & 6081.432 & 3 \\
\hline $6097.60 .$. & 2 & B & & & 6097.633 & 3 & 6097.839 & 5 \\
\hline & & & & & 6112.70 & 0 & 6112. 961 & 1 \\
\hline $6129.98 . \ldots$ & $3, \mathrm{H},+$ & B & & & $\cdots$ & & …......... & . \\
\hline $6178.85 \ldots$. & 1 & D & & & ............ & ... & ............. & .... \\
\hline $6179.80 \ldots$ & 1 & B & & & ............. & $\ldots$ & .............. & .. \\
\hline & & & & & 6204.51 & 0 & 6204.682 & 3 \\
\hline $6214.00 \ldots$ & 2 & B & & & n.......... & $\cdots$ & .......... & . \\
\hline $6221.45 \ldots$ & 3 & $\mathbf{A}$ & & & ............ & $\cdots$ & (n.......... & . \\
\hline & & & & & 6332.220 & 3 & 6332.395 & 5 \\
\hline & & & & & 6345.955 & 2 & 6346.098 & 4 \\
\hline & & & & & 6388.324 & 2 & 6388.522 & 2 \\
\hline & & & & & 6392.175 & 3 & 6392.391 & 3 \\
\hline & & & & & 6405.406 & 2 & 6405.611 & 3 \\
\hline $6415.93 \ldots$ & $1, \mathbf{H}$ & B & & & & & .... & . \\
\hline $6454.95 . \ldots \ldots \ldots \ldots \ldots$ & 2 & B & & & ............ & ... & ............ & a...... \\
\hline $6490.25 \ldots \ldots \ldots \ldots \ldots$ & 2 & B & & & ... & & 6495.173 & ........ \\
\hline $6503.03 .$. & $1, \mathbf{E}$ & C & & & & & n........ & $\cdots \cdots \cdot$ \\
\hline & & & & & 6505.404 & 1 & 6505.657 & 3 \\
\hline $6517.10 .$. & 2 & B & & & & ... & …….... & ….... \\
\hline & & & & & 6558.150 & 3 & 6558.353 & 5 \\
\hline & & & & & 6564.052 & 3 & 6564.269 & 5 \\
\hline $6611.48 . \ldots \ldots \ldots .$. & 3 & B & & & 6611.411 & 3 & 6611.146 & 3 \\
\hline $6634.63 \ldots \ldots \ldots \ldots \ldots$ & $2, \mathbf{H}$ & B & $\cdots$ & & n......... & ......... & (........... & (......... \\
\hline $6640.55 \ldots \ldots \ldots \ldots \ldots \ldots$ & $1, \mathbf{H}$ & B & & & 6648.134 & $\begin{array}{r}\cdots \\
2\end{array}$ & 6648.344 & 4 \\
\hline
\end{tabular}

a Eder and Valenta. Atlas Typsicher Spectren, Wien; rgrr.

b Schippers, Zeits. f. Wiss. Phot., 11, p. 235; 1912-13.

c Kretzer, Zeits. f. Wiss. Phot., 8, p. 56; 19 ro.

d Given by Kretzer as spark ines. 
TABLE 8-Continued

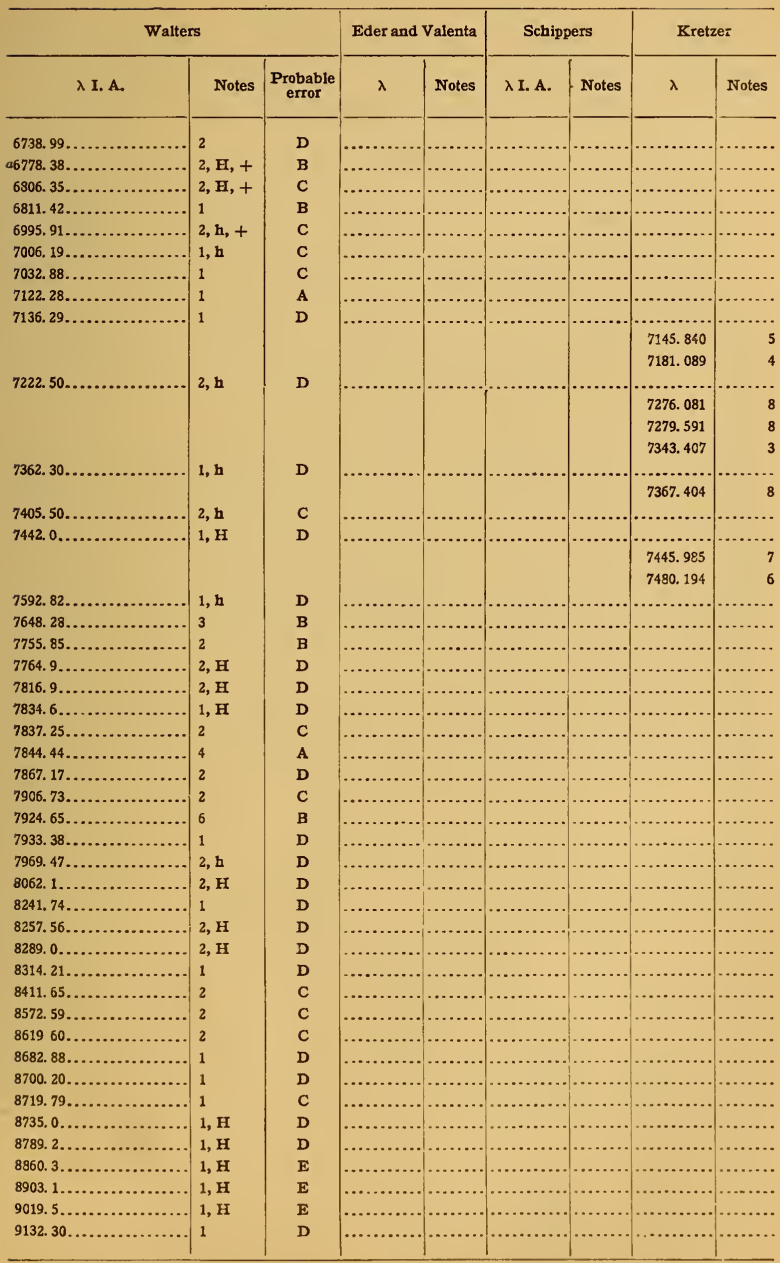

a Given by Kretzer as spark lines. 


\section{TIN}

Metallic tin was used in copper or graphite electrodes for the production of the arc spectrum. Sodium and potassium were observed as impurities, and by the same token the line at 7800.282 A observed by Arnolds may be due to rubidium.

TABLE 9.-Tin

\begin{tabular}{|c|c|c|c|c|c|c|c|c|}
\hline \multicolumn{3}{|c|}{ Walters } & \multicolumn{2}{|c|}{ Arnolds $a$} & \multicolumn{2}{|c|}{$\frac{\text { Kayser and }}{\text { Runge } b}$} & \multicolumn{2}{|c|}{$\begin{array}{l}\text { Eder and } \\
\text { Valenta } c\end{array}$} \\
\hline$\lambda I . A$. & Notes & $\begin{array}{c}\text { Probable } \\
\text { error }\end{array}$ & $\lambda$ I. A. & Notes & $\lambda$ & Notes & $\lambda$ & Notes \\
\hline $5631.70 \ldots$ & 8 & $\mathbf{A}$ & 5631.696 & & 5631.91 & 5 & 5631.89 & 5 \\
\hline $5753.61 \ldots$ & 3 & B & ....... & & & & & .. \\
\hline $5761.77 \ldots \ldots \ldots$ & 2 & $\mathbf{A}$ & & & & & & \\
\hline $5801.79 \ldots$ & 1 & $\mathbf{A}$ & $\ldots . .$. & & & & & \\
\hline $5925.48 \ldots \ldots \ldots \ldots \ldots . . . .$. & 4 & B & & & & & & \\
\hline $5934.72 \ldots$ & $2, \mathrm{H}$ & D & & & & & & $\cdots$ \\
\hline $5970.30 \ldots$ & 5 & $\mathbf{A}$ & & & & & & \\
\hline $6011.08 \ldots$ & 2,h & D & & & & & & .... \\
\hline $6037.70 .$. & 5 & $\mathbf{B}$ & $\ldots . .$. & & & & & ... \\
\hline $6054.90 \ldots$ & 5 & D & ........ & & & & & \\
\hline $6060.75 \ldots \ldots \ldots \ldots$ & 2 & C & ........... & & & & & .... \\
\hline $6068.94 \ldots$ & $8, \mathrm{~h}$ & C & 6069.08 & $2, \mathrm{u}$ & & & & \\
\hline $6073.52 \ldots \ldots \ldots \ldots \ldots . . . . . .$. & $4, \mathrm{~h}$ & C & 6073.55 & $1, \mathrm{ul}$ & & & & .... \\
\hline $6149.67 \ldots \ldots \ldots \ldots . . . .$. & 6 & $\mathbf{B}$ & 6149.620 & 3 & 6149.81 & $2, v$ & & ... \\
\hline $6154.60 \ldots \ldots \ldots$. & 5 & $\mathbf{B}$ & & & & & & \\
\hline $6171.49 \ldots \ldots \ldots \ldots$ & 4 & B & & & & & & ... \\
\hline $6203.62 \ldots \ldots \ldots . .$. & 3 & B & & & & & & \\
\hline $6275.78 .$. & 2 & B & & & & & & .. \\
\hline $6310.83 \ldots \ldots \ldots \ldots$ & 4 & c & $\cdots$ & & & & & $\cdots$ \\
\hline $6254.36 \ldots \ldots \ldots \ldots$ & 2 & $\mathbf{B}$ & & & & & & \\
\hline $6444.83 \ldots \ldots \ldots \ldots \ldots \ldots \ldots$ & 2,H & $\mathbf{D}$ & & & & & & \\
\hline $6453.58 \ldots \ldots \ldots \ldots \ldots \ldots$ & 3 & A & .... & & & & & $\cdots$ \\
\hline $6462.36 \ldots \ldots \ldots$ & $2,+, \mathrm{Ca} ?$ & D & & & & & & \\
\hline $6844.20 \ldots \ldots \ldots \ldots \ldots \ldots$ & 2 & $\mathbf{B}$ & & & & & & \\
\hline $6909.29 \ldots \ldots \ldots \ldots \ldots \ldots$ & $1, \mathrm{H}$ & D & & & & & & ... \\
\hline $6924.72 \ldots \ldots \ldots \ldots \ldots . . . . . . .$. & 1,H & $\mathbf{E}$ & & & & & & $\cdots$ \\
\hline $7685.29 \ldots \ldots \ldots \ldots \ldots \ldots$ & 1 & $\mathbf{A}$ & ............ & $\cdots$ & & & & .... \\
\hline $7754.94 \ldots \ldots \ldots \ldots \ldots \ldots$ & 2 & $\mathbf{A}$ & (............ & ........ & & & & ......... \\
\hline & & & 7800.282 & 1 & & & & \\
\hline $7808.25 \ldots \ldots \ldots \ldots$ & 1 & $E$ & ........ & & & & & .......... \\
\hline $8100.42 \ldots \ldots \ldots \ldots \ldots \ldots . . . . . . .$. & 2 & $\mathrm{D}$ & & & & & & (........ \\
\hline $8114.06 \ldots \ldots \ldots \ldots \ldots \ldots$ & 7 & C & & & & & & . \\
\hline $8121.42 \ldots \ldots \ldots \ldots \ldots \ldots . . . . . . . .$. & 2,E & C & & & & & & $\cdots$. \\
\hline $8249.6 \ldots \ldots \ldots \ldots \ldots \ldots . . . . . . .$. & $1, \mathrm{H}$ & $D$ & $\cdots .$. & $\cdots$ & & & & ....... \\
\hline $8338.0 \ldots \ldots \ldots \ldots \ldots \ldots . . . . . .$. & $1, \mathrm{H}$ & D & & & & & & \\
\hline $8345.38, \ldots \ldots \ldots \ldots \ldots \ldots$ & $1, \mathrm{~h}$ & D & & & & & $\ldots$. & ....... \\
\hline $8349.35 \ldots \ldots \ldots \ldots \ldots \ldots$. & 3 & $\mathrm{D}$ & ........... & ......... & & $\cdots$ & $\cdots$ & ......... \\
\hline $8357.03 . \ldots \ldots \ldots \ldots \ldots \ldots$ & 4 & C & ...... & . & & & & ......... \\
\hline $8391.27 \ldots \ldots \ldots \ldots \ldots \ldots . . . . .$. & 2 & D & …...... & ...... & & & & ...... \\
\hline $8422.77 \ldots \ldots \ldots \ldots \ldots \ldots$ & $3, \mathrm{~h}$ & $\mathbf{D}$ & (.......... & ........ & & & & \\
\hline $8457.6 \ldots$ & 2,H & D & & & & & & \\
\hline
\end{tabular}

a Arnolds, Zeits. f. Wiss. Phot., 13, p. $3 \mathrm{r}_{3} ; \mathrm{x}^{2} \mathrm{r}_{3}-\mathrm{I} 4$.

$\checkmark$ Kayser and Runge, Wied. Ann., 52, p. 93 ; 1894.

$c$ Eder and Valenta, Wien, Ber., 119, Ila, D. 519; x9ro. 
TABLE 9-Continued

\begin{tabular}{|c|c|c|c|c|c|c|c|c|}
\hline \multicolumn{3}{|c|}{ Walters } & \multicolumn{2}{|c|}{ Randall $a$} & \multicolumn{2}{|c|}{$\begin{array}{c}\text { Kayser and } \\
\text { Runge }\end{array}$} & \multicolumn{2}{|c|}{$\begin{array}{c}\text { Eder and } \\
\text { Valenta }\end{array}$} \\
\hline$\lambda$ I. A. & Notes & $\begin{array}{c}\text { Probable } \\
\text { error }\end{array}$ & $\lambda$ & Notes & $\lambda$ & Notes & $\lambda$ & Notes \\
\hline $8552.60 .$. & 7 & B & 8554.7 & 12 & & & & \\
\hline $8649.2 \ldots \ldots$. & $1, \mathrm{H}$ & D & . & & & & & \\
\hline $8681.4 \ldots \ldots \ldots \ldots$ & $1, \mathrm{H}$ & D & & ... & & & & \\
\hline $8708.9 \ldots$ & $1, \mathrm{H}$ & D & 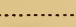 & .... & & & & \\
\hline $8886.61 \ldots \ldots \ldots . . .$. & 1 & D & ........... & .... & & & & \\
\hline $8908.11 \ldots \ldots \ldots . .$. & . $1, \mathrm{~h}$ & $\mathbf{E}$ & & & & & & \\
\hline $9018.9 \ldots \ldots . .$. & $1, \mathrm{~h}$ & D & .... & ... & & & & \\
\hline $9023.1 \ldots \ldots \ldots \ldots$ & $1, \mathrm{~h}$ & D & 9023.2 & 8 & & & & \\
\hline $9146.3 \ldots \ldots \ldots \ldots$ & $1, \mathrm{H}$ & D & ......... & & & & & \\
\hline
\end{tabular}

a Randall, Astrophys. J., 31, p. I; $191 \mathrm{rI}$ (bolometer measurements to 13022 A).

\section{ZINC}

Chemically pure zinc was burned in copper electrodes and also with a ro $\mathrm{mm}$ zinc rod as the positive electrode and copper as the negative electrode. Lead, cadmium, sodium, and potassium were found as impurities. Of the "new" lines given by Eder from 7026 to $8256 \mathrm{~A}$, 18 correspond to the stronger vanadium lines in this region; 7664.84 and $7698.93 \mathrm{~A}$, since they both appear, look suspiciously like potassium, and $7625 \mathrm{~A}$ may be due to nickel. This accounts for all except 7932.95 and $7937.93 \mathrm{~A}$ which are in all probability also due to some impurity. Eder admits that "these red and infra-red lines of zinc appear to be but slightly characteristic and are scarcely suited for spectral analytical indications of the metal."

TABLE 10.-Zinc

\begin{tabular}{|c|c|c|c|c|c|c|c|c|c|c|}
\hline \multicolumn{3}{|c|}{ Walters } & \multicolumn{2}{|c|}{ Eder and Valenta $a$} & \multicolumn{2}{|c|}{ Wiedmann $b$} & \multicolumn{2}{|c|}{ Paschen $c$} & \multicolumn{2}{|c|}{ Lehmann $d$} \\
\hline$\lambda$ I. A. & Notes & $\begin{array}{c}\text { Probable } \\
\text { error }\end{array}$ & $\lambda$ & Notes & $\lambda$ & Notes & $\lambda$ & Notes & $\lambda$ & Notes \\
\hline & & & & & 5654.48 & 1 & ….... & & & \\
\hline $5772.64 \ldots$ & 4,2 & D & & & 5772.33 & 5 & 5772.218 & 10 & & \\
\hline $5775.94 \ldots$ & $2, L$ & D & & & 5775.75 & 4 & 5775.645 & 8 & & \\
\hline $5777.81 \ldots$ & $1, L$ & D & & & 5777.36 & 3 & 5777.240 & 6 & & \\
\hline $5894.38 \ldots$ & 2 & B & & & 5894.65 & 6 & 5894.53 & 10 & & \\
\hline & & & & & & & 5937.93 & 3 & & \\
\hline & & & & & 6022 & 1 & $\cdots$ & & & \\
\hline & & & & & 6102.38 & 2 & & & & \\
\hline $6183.97 \ldots$ & $5, \mathrm{~h}, \mathrm{v}$ & D & & & ........... & $\cdots$ & ........... & ... & .... & $\cdots$ \\
\hline $6214.62 \ldots$ & 1 & D & & & 6214.86 & 5 & 6214.89 & 7 & & \\
\hline $6237.82 \ldots$ & $3, \mathrm{~h}$ & C & & & 6238.20 & 6 & 6238.21 & 8 & & \\
\hline
\end{tabular}

a Eder and Valenta, Wien, Ber., I18, IIa, p. I077; 1909.

$b$ Weidmann, Ann. d. Phys., 35, p. 660; rgrx.

c Paschen, Ann. d. Phys., 29, p. 625; 1909.

d Lehmann, Ann. d. Phys., 39, p. 53; 19 ra. 
TABLE 10-Continued

\begin{tabular}{|c|c|c|c|c|c|c|c|c|c|c|}
\hline \multicolumn{3}{|c|}{ Walters } & \multicolumn{2}{|c|}{ Eder and Valenta } & \multicolumn{2}{|c|}{ Wiedmann } & \multicolumn{2}{|c|}{ Paschen } & \multicolumn{2}{|c|}{ Lehmann } \\
\hline$\lambda$ I. A. & Notes & $\begin{array}{c}\text { Probable } \\
\text { error }\end{array}$ & $\lambda$ & Notes & $\lambda$ & Notes & $\lambda$ & Notes & $\lambda$ & Notes \\
\hline $6239.14 \ldots .$. & 2 & C & & & 6239.46 & 3 & 6239.43 & 6 & & \\
\hline \multirow[t]{2}{*}{ a $6362.345 .}$. & 10 & (.......... & 6362.58 & 8 & 6362.58 & 10 & 6362.58 & 20 & 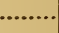 & $\cdots$ \\
\hline & & & & & & & 6471.19 & 4 & & \\
\hline \multirow[t]{2}{*}{$6478.84 \ldots \ldots$} & $2, \mathrm{~h}$ & D & & $\cdots$ & 6479.50 & 5 & 6479.37 & 8 & & \\
\hline & & & & & & & 6914.15 & 2 & & \\
\hline $6928.69 \ldots$. & $8, L$ & $\mathrm{D}$ & 6929.0 & 4 & 5928.54 & 8 & 6928.582 & 8 & ........ & $\cdots$ \\
\hline $6938.77 \ldots .$. & $6, L$ & D & 6939.2 & 3 & 6938.68 & 6 & 6938.733 & 6 & 6937.2 & 2 \\
\hline \multirow[t]{10}{*}{$6943.55 \ldots .}$. & $3, \mathrm{~L}$ & D & 6943.9 & 2 & 6943.46 & 4 & 6943.474 & 4 & $\cdots$ & \\
\hline & & & \multicolumn{2}{|c|}{ Eder $b$} & & & & & & \\
\hline & & & $\lambda$ I. A. & Notes & & & & & & \\
\hline & & & 7026.07 & 4 & & & & & & \\
\hline & & & 7264.22 & 4 & & & & & 7234.9 & \\
\hline & & & 7338.88 & 4 & & & & & & \\
\hline & & & 7356.45 & 4 & & & & & & \\
\hline & & & 7358.54 & 4 & & & & & & \\
\hline & & & 7361.33 & 3 & ..... & & & & & \\
\hline & & & 7363.11 & 3 & .. & & & & & \\
\hline $7468.44 \ldots$ & 1 & D & .............. & & & & & & & \\
\hline $7478.71 \ldots$ & 2 & C & ............ & $\cdots$ & 7479.03 & 6 & & & & \\
\hline & & & 7578.75 & 3 & ...... & & & & & \\
\hline & & & 7624.77 & 3 & $\cdots \cdots$ & & & & & \\
\hline & & & & & & & & & 7675.1 & 4 \\
\hline & & & 7664.84 & 4 & & & & & & . \\
\hline & & & 7698.93 & 4 & $\ldots .$. & & & & & *. \\
\hline $7799.04 \ldots$. & $4, \mathrm{~h}, \mathrm{v}$ & D & ............. & $\cdots$ & 7799.62 & 6 & & & & .. \\
\hline & & & 7932.95 & 2 & $\cdots \cdots$ & & & & & \\
\hline & & & 7937.93 & 2 & & & & & 8005.2 & 3, u \\
\hline & & & & & & & & & 8112.1 & $4, u$ \\
\hline & & & 8116.74 & 4 & & & & & & ....... \\
\hline & & & 8144.57 & 2 & & & & & & ........ \\
\hline & & & 8161.01 & 3 & & & & & & $\ldots$ \\
\hline & & & 8186.65 & 2 & $\cdots$ & & 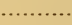 & & & .... \\
\hline & & & 8187. 38 & 1 & & & & & & \\
\hline & & & 8198.85 & 2 & . & & & & & $\cdots$ \\
\hline & & & 8202. 91 & 2 & ..... & & .... & & & ....... \\
\hline & & & 8241.50 & 1 & & & & & & \\
\hline & & & 8253.46 & 1 & $\cdots$ & & $\cdots$ & & & \\
\hline & & & 8255.76 & 1 & 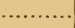 & & & & & \\
\hline
\end{tabular}

a Interferometer measurement, vibrator in vacuo, Fabry and Perot, Comptes Rendus, 130, p. 242; 1900. $b$ Eder, Wien, Ber., 124, IIa, p. 120; 1915. 


\section{DISCUSSION}

A comparison of the wave lengtis measured for the same element by different observers shows several important facts. In the first place impurity and spurious lines must be looked for very carefully. The determination, in a given spectral region, of the wave lengths of related or contaminating elements makes increasingly more certain the lines belonging to each particular element, and only when the spectra of all the elements are intcrcompared on the same basis can the true standard spectrum of eacli element be arrived at. Spurious lines due to false spectra given by gratings or to unrecognized overlapping spectral orders are responsible for some of the divergences in spectroscopic ditta. Careful examination of the optical performance of gratings should remove the first, and improvements in ray filters and plotographic plates are necessary to reduce errors of the second kind. It is important to describe the source of light and specify (he olsscrving conditions since the spectrum of each clement in gencral is different under various operating conditions (are or spark, cither in air, vácumm, or under pressure, etc.) and appreciably differesu spectra are obtained even from the sime source if light from different parts of it is eximined (pole effect, electrode lines, ete.). The aceuracy possible in wave-length ineasurements from the direct photography of normal spectra exceeds that of phosploor plotography and bolometer observations, but at the present tume the photographic method is limited to waves shorter than about $10000 \mathrm{~A}$. The development of a plotographic plate sensitive further in to the infra-red is much to be desired.

\section{SUMMARY}

The are spectra of 10 metals, silver, aluminum, gold, bismuth, cadinium, mercury, lead, antimony, tin, and zinc, were photographed in the red and infra-red on plates bathed with pinacyanol and dicyanin. These elements have relatively few strong lines in this region when compared with the spectra of iron, cobalt, and nickel observed under the same conditions.

In conclusion, acknowledgment is made to Prof. O. M. Stewart and to Dr. W. F. Aleggers for their interest and encouragement in this work.

Wasmington, December 9, 1920. 